\section{Surgical outcomes of 25-gauge pars plana vitrectomy for diabetic tractional retinal detachment}

MS Dikopf ${ }^{1}, \mathrm{KH}$ Patel $^{1}$, VJ Setlur and JI Lim

\begin{abstract}
Purpose To evaluate the outcomes and complications of 25-gauge (G) pars plana vitrectomy (PPV) for repair of diabetic tractional retinal detachment (TRD). Methods Retrospective review of consecutive, single-surgeon 25-G PPV cases between July 2007 and July 2014. Seventy eyes from 55 patients were operated on for diabetic TRD; all eyes were tamponaded with sulfur hexafluoride, octofluoropropane, silicone oil, or balanced salt solution. Mean age at surgery was 47.7 years (range 23-76 years), and mean length of follow-up was 713 days (range 90-2368 days; median 671 days). Primary outcomes included bestcorrected visual acuity (BCVA), intraocular pressure (IOP), anatomic success, redetachment, and endophthalmitis.
\end{abstract}

Results Preoperatively, 49 eyes (70\%) had a concurrent rhegmatogenous component (8 of which also had proliferative vitreoretinopathy (PVR)). Mean BCVA improved from logarithm of the minimal angle of resolution $1.59(20 / 800$, SD 0.88$)$ to 0.68 postoperatively (20/100, 0.77), $P$-value $<0.001$. Mean IOP increased from 15.9 to $20 \mathrm{~mm} \mathrm{Hg} 1$ day after surgery. Elevated postoperative IOP $(\geq 22 \mathrm{~mm} \mathrm{Hg}$ ) occurred in 25 eyes, and low IOP ( $\leq 5 \mathrm{~mm} \mathrm{Hg}$ ) occurred in 2 eyes. Primary reattachment was achieved in 63 eyes $(90 \%)$, and final anatomical success occurred in 69 eyes $(99 \%)$. There were no cases of endophthalmitis.

Conclusions Twenty-five-G PPV repair was safe and effective in the repair of diabetic TRD, including eyes with a combined rhegmatogenous detachment or PVR. Gas, silicone oil, and balanced salt solution tamponading agents all proved to be efficacious in this surgical population. Eye (2015) 29, 1213-1219; doi:10.1038/eye.2015.126; published online 17 July 2015

\section{Introduction}

Tractional retinal detachment (TRD) is a major cause of vision loss in patients with proliferative diabetic retinopathy (PDR). ${ }^{1,2}$ In about $5-10 \%$ of cases of retinal detachment, anatomical and visual prognoses are complicated by proliferative vitreoretinopathy (PVR). ${ }^{3,4}$ Surgical management of diabetic TRD, with or without PVR, may often be challenging.

Repair of complicated vitreoretinal disease, such as diabetic TRD, has conventionally been accomplished via 20-gauge $(\mathrm{G})$ or $23-\mathrm{G}$ pars plana vitrectomy (PPV) systems. However, the 25-G pars plana vitrectomy (PPV) system, first described by Fujii and colleagues in 2002, ${ }^{5}$ offers many advantages over 20- or 23-G PPV. These include reduced operative times, reduced postoperative inflammation, faster visual recovery, increased patient comfort, reduced rate of intraoperative retinal break formation, avoidance of conjunctival dissection and limbal stem cell damage, and less induced corneal astigmatism. $^{5-12}$

Upon its advent, 25-G PPV was applied towards treating macular holes, idiopathic epiretinal membranes, refractory macular edema, and non-clearing vitreous hemorrhage. ${ }^{13}$ There were concerns that fragility or inadequate variety of instrumentation may limit its usefulness in more complicated surgical disease. ${ }^{14,15}$ However, instrumentation has expanded, ${ }^{16}$ and stiffer $25-\mathrm{G}$ instrumentation has led to successful outcomes of 25-G PPV for a wide variety of vitreoretinal pathologies. ${ }^{6}$

Given the benefits of 25-G systems, several studies have investigated its use in the treatment of complicated retinal detachment, including diabetic TRD; comparable outcomes and complication rates between 25-G PPV and 20-G or 23-G systems have been found. $6,7,17-20$ However, these studies included limited
Retina Service, UIC Department of Ophthalmology and Visual Sciences, Illinois Eye and Ear Infirmary, University of Illinois Hospital \& Health Sciences System, Chicago, IL, USA

Correspondence: JI Lim, Retina Service, UIC Department of Ophthalmology and Visual Sciences, Illinois Eye and Ear Infirmary, University of Illinois Hospital \& Health Sciences System, 1855 W. Taylor Street, Suite 2.50, Mail Code 648, Chicago, IL 60611, USA

Tel: +1 312413 0704;

Fax +1 3124137929 .

E-mail: jennylim@uic.edu

${ }^{1}$ These authors contributed equally to this work.

Received: 6 January 2015 Accepted in revised form: 26 May 2015

Published online:

17 July 2015

Preliminary data from this series was presented at the 2013 Annual Meeting of the Association for Research in Vision and Ophthalmology. 
numbers of TRD eyes and only evaluated silicone oil or octofluoropropane (C3F8) tamponades. This study aims to evaluate the effectiveness of 25-G PPV and a variety of tamponade agents in a large population of eyes with diabetic TRD, many of which also had combined rhegmatogenous retinal detachments (RRD) or PVR.

\section{Materials and methods}

We conducted a retrospective review of the medical records of all surgical cases performed by a single vitreoretinal surgeon (JIL) at the University of Illinois Hospital, between July 2007 and July 2014. The study was conducted in compliance with the Health Insurance Portability and Accountability Act and was approved by the Institutional Review Board at the University of Illinois, Chicago. Eyes that underwent 25-G PPV for primary repair of a diabetic TRD were eligible for inclusion; patients with $<3$ months of postoperative follow-up, or patients with other proliferative vitreoretinopathy disease (such as proliferative sickle cell retinopathy) were excluded. No patients underwent preoperative treatment with anti-VEGF agents.

PPV was performed using the Alcon Accurus or Alcon Constellation 25-G vitrectomy machines (Alcon Laboratories, Inc., Fort Worth, TX, USA), with a maximum cut rate of 5000 cuts per min and maximum vacuum rate of $650 \mathrm{~mm} \mathrm{Hg}$. In cases that required bimanual technique for dissection of extensive fibrovascular membranes, an illuminated $25-\mathrm{G}$ pick or a $25-\mathrm{G}$ self-retaining chandelier illumination fiber was utilized. All cases underwent vitrectomy and vitreous base shaving using a wide-angle viewing system and scleral depression during the vitreous base dissection. Perfluro-n-octane (PFO) liquid was used as needed. Endolaser photocoagulation was applied for treatment of neovascularization or to barricade retinal breaks. Fluid-air exchange followed by injection of sulfur hexafluoride $\left(\mathrm{SF}_{6}\right), \mathrm{C} 3 \mathrm{~F} 8$, silicone oil, or balanced salt solution (BSS) was performed to tamponade retinal breaks or detachments. The choice of tamponade was determined by the extent of detachment, amount of traction, existence of a rhegmatogenous component and, in rare cases, the patient's preoperative preference for silicone oil instead of gas.

In patients with a preoperative visually significant cataract, cataract extraction and implantation of an acrylic, foldable intraocular lens was performed via pars plana lensectomy or phacoemulsification through a clear corneal incision.

All sclertomies in this series were sewn to prevent against postoperative hypotony or infection, as discussed later.

Primary outcome measures included postoperative best-correct Snellen visual acuity (BCVA), postoperative intraocular pressure (IOP) measured by Goldmann applanation tonometry or a Tono-pen XL applanation tonometer (Reichert Technologies, Depew, NY, USA), and rates of postoperative complications including redetachment, vitreous hemorrhage, epiretinal membrane formation, ocular hypertension or hypotony, and endophthalmitis.

BCVA was measured using the standard Snellen visual acuity chart and converted to the logarithm of the minimal angle of resolution (LogMAR) visual acuity. ${ }^{21}$ Peak BCVA at any time during the postoperative period was used to represent the postoperative LogMAR visual acuity. Preoperative and postoperative LogMAR values were compared with a paired, two-tailed $t$-test, and a $P$-value $<0.05$ was considered statistically significant.

Outcomes for all eyes, as well as per form of tamponade were performed. Given variability in location and type of retinal detachments, subgroup analysis was also performed in regards to macular involvement, existence of a rhegmatogenous component, and existence of PVR.

\section{Results}

\section{Patient demographics}

Seventy eyes from 55 patients were included in this study (Table 1). The mean age of all patients was 47.7 years (range, 23-76 years) and mean length of follow-up was 713 days (range, 90-2368 days; median 671 days). Of note, 6 of our 55 patients were lost to follow-up between 3 and 6 months after surgery. Four of them were tamponaded with SF6, 1 with C3F8 and 1 had BSS; none had PVR. Mean follow-up excluding these patients was 775 days (range 204-2368 days; median 730 days). All 55 patients were included for data analysis.

Table 1 Patient characteristics

\begin{tabular}{lc}
\hline Number of eyes & 70 \\
Number of patients & 55 \\
Male/female & $23 / 32(42 \% / 58 \%)$ \\
Mean age at surgery (years) & 47.7 \\
Mean length of follow-up (days) & 713 \\
Median lenth of follow-up (days) & 671 \\
Mean preoperative IOP (mm Hg) & 15.9 \\
Mean postoperative day 1 IOP (mm Hg) & 20 \\
Eyes phakic preoperatively & $66(94 \%)$ \\
Eyes with lens extracted in combination & $10(14 \%)$ \\
with PPV & \\
Eyes with PFO used intraoperatively & $15(21 \%)$ \\
Eyes with preoperative vitreous & $53(76 \%)$ \\
hemorrhage present & \\
Mean operative time (min) & 118
\end{tabular}

Abbreviations: IOP, intraocular pressure; PFO, perfluoro-n-octane; PPV, pars plana vitrectomy. 
Table 2 Visual acuity outcomes and anatomical success per form of tamponade

\begin{tabular}{lcccccc}
\hline $\begin{array}{l}\text { Form of } \\
\text { tamponade }\end{array}$ & $\begin{array}{c}\text { Number } \\
\text { of eyes }\end{array}$ & $\begin{array}{c}\text { Mean preoperative visual } \\
\text { acuity (LogMAR) }\end{array}$ & $\begin{array}{c}\text { Mean peak postoperative } \\
\text { visual acuity (LogMAR) }\end{array}$ & P-value & $\begin{array}{c}\text { Eyes with primary } \\
\text { reattachment achieved }\end{array}$ & $\begin{array}{c}\text { Eyes with final anatomical } \\
\text { success achieved }\end{array}$ \\
\hline SF $_{6}$ Gas & 22 & $1.48(\sim 20 / 600)$ & $0.51(\sim 20 / 60)$ & $<0.001$ & $20(91 \%)$ & $21(96 \%)$ \\
C3F8 Gas & 22 & $1.49(\sim 20 / 600)$ & $0.95(\sim 20 / 175)$ & $<0.05$ & $18(82 \%)$ & $22(100 \%)$ \\
BSS & 21 & $1.69(\sim 20 / 100)$ & $0.42(\sim 20 / 50)$ & $<0.001$ & $21(100 \%)$ & $21(100 \%)$ \\
Silicone Oil & 5 & $2.15(\sim 20 / 3000)$ & $1.53(\sim 20 / 700)$ & 0.37 & $4(80 \%)$ & $5(100 \%)$ \\
Overall & 70 & $1.59(\sim 20 / 800)$ & $0.68(\sim 20 / 100)$ & $<0.001$ & $63(90 \%)$ & $69(99 \%)$ \\
\hline
\end{tabular}

Abbreviations: BSS, balanced salt solution; LogMAR, logarithm of the minimum angle of resolution.

Mean preoperative IOP was $15.9 \mathrm{~mm} \mathrm{Hg}$ (range, 7-30 $\mathrm{mm} \mathrm{Hg}$ ); preoperative IOP was unavailable for one eye. Preoperative vitreous hemorrhage was present in 53 eyes. Four eyes were pseudophakic preoperatively, while the remaining 66 eyes were phakic-10 had a visually significant cataract that required extraction during the primary TRD repair.

\section{Operative findings}

Forty-nine out of 70 eyes (70\%) had a combined rhegmatogenous and tractional retinal detachment, and 8 of these eyes (11\%) also had PVR. During surgery, five eyes had a sclerotomy site enlarged to 20-G. In four eyes this was performed secondary to instrumentation; three required the use of a 20-G fragmentor for lensectomy, whereas another required the use of a 20-G endolaser because the 25-G model was unavailable. For the fifth eye, a sclerotomy was enlarged to acquire a sample of neovascularization of the disc for research purposes. For all eyes with an enlarged sclerotomy, a 20-25-G plug was utilized to allow for continued use of 25-G instrumentation. PFO liquid was used for intraoperative flattening of detached retina in 15 eyes. All eyes were ultimately tamponaded with $\mathrm{SF}_{6}$ (22 eyes), C3F8 (22), BSS (21), or silicone oil (5).

Mean operative time was $118 \mathrm{~min}$ (range, 52-270 min); operative time was unavailable for six eyes.

\section{Surgical outcomes}

For all eyes, mean preoperative BCVA improved from LogMAR 1.59 (20/800, SD 0.88, 95\% confidence interval (CI) $1.38-1.80)$ to $0.68(20 / 100,0.77,0.50-0.86)$ after surgery, $P<0.001$. Postoperative BCVA improvements were found in all forms of tamponade. Eyes with $\mathrm{SF}_{6}$ gas improved from LogMAR 1.48 (20/600, SD 0.64, CI $1.17-1.76)$ to $0.51(20 / 60,0.65,0.30-0.72), P<0.001$. Eyes with C3F8 gas improved from 1.49 (20/600, 0.99, $1.07-1.90)$ to $0.95(20 / 175,0.93,0.56-1.34), P<0.05$. Eyes with BSS tamponade improved from 1.69 (20/1000, 0.96, $1.28-2.1)$ to $0.42(20 / 50, .40,0.25-0.59), P<0.001$. Eyes that underwent silicone oil tamponade had the worst mean preoperative BCVA of 2.15 (20/3000, 0.98, 1.29-3.01), and improved to a mean of 1.53 (20/700,1.10, 0.57-2.49), $P=0.37$ (Table 2). Of note, four eyes, one in each tamponade group, had pre- or postoperative visual acuities that were light perception or worse, and therefore could not be converted to LogMAR. ${ }^{21}$ Three eyes had a preoperative visual acuity of light perception; two improved postoperatively to $20 / 400$, whereas the other remained at light perception. A fourth eye worsened from hand motion to light perception, due to neovascular glaucoma and recurrent, dense vitreous hemorrhage.

As shown in Table 3, 49 eyes with a combination tractional and rhegmatogenous detachment improved from a preoperative mean LogMAR BCVA of 1.54 $(20 / 700,0.85,1.30-1.80)$ to 0.80 (20/125, 0.87, 0.56-1.04), $P<0.001$. Of the 21 eyes with solely a TRD, Snellen acuity improved from a mean of $1.7(20 / 1000,0.95,1.29-2.11)$ to $0.42(20 / 50,0.40,0.25-0.54), P<0.001$. Thirty-nine patients had a detachment involving the macula; preoperative BCVA was $1.61(20 / 800,0.82,1.35-1.87)$ and improved to 0.86 (20/145, 0.76, 0.62-1.10), $P<0.001$. Thirty-one patients were operated on prior to macular involvement; among these eyes, vision improved from a mean of $1.56(20 / 725,0.96,1.22-1.90)$ to $0.47(20 / 60,0.74$, $0.21-0.73), P<0.001$. Of the eyes that a combined TRD and RRD, 8 also had pre-existing PVR; in this group, preoperative mean BCVA of 2.3 (20/4000, 0.76, 1.77-3.06) improved to $1.88(20 / 1500,1.25,1.01-2.75), P=0.31$.

Overall, primary reattachment was achieved in 63 eyes (90\%). Six eyes redetached, and 5 were reattached with a total of six additional surgeries; final anatomical success was therefore achieved in 69 eyes (99\%). One eye was noted to have a macular sparing TRD at the last visit; vision was hand motion and limited by severe retinal ischemia, and the patient declined further surgical intervention.

\section{Postoperative complications}

Mean post-operative day-1 IOP was $20 \mathrm{~mm} \mathrm{Hg}$ (range, 2-45 mm Hg). Postoperative day-1 IOP was unavailable for five eyes, however, all patients were closely followed and postoperative week-1 IOP values were available (week-1 values were not substituted into the data 
analysis). Elevated IOP ( $\geq 22 \mathrm{~mm} \mathrm{Hg}$ ) on postoperative day 1 occurred in 25 eyes. Specifically, this occurred in 10 eyes with $\mathrm{SF}_{6}$ gas (45\% of eyes in this group), 9 eyes with C3F8 gas (41\%), 5 eyes with BSS (24\%), and 1 eye with silicone oil (20\%). Two of these $\mathrm{SF}_{6}$ eyes had a combined lensectomy ( $50 \%$ of total combined lensectomies in this group), one of the C3F8 eyes (100\%), two of the BSS eyes $(67 \%)$, and one of the silicone oil eyes (50\%). Most cases of postoperative ocular hypertension were adequately managed with topical medications, however, few complicated cases occurred in each group. One of the eyes with $\mathrm{SF}_{6}$ was noted to have an IOP of $24 \mathrm{~mm} \mathrm{Hg}$ preoperatively, which rose to 38 on postoperation day 1 ; this was initially resistant to topical medications necessitating an anterior chamber tap, and intraocular pressures were thereafter maintained in the teens with topical medicine. However, 2 months after surgery, this eye was found to have a pressure of $44 \mathrm{~mm} \mathrm{Hg}$ secondary to neovascular glaucoma, requiring transcleral diode laser cyclophotocoagulation. Further, one eye with C3F8 was found to have an IOP of $40 \mathrm{~mm} \mathrm{Hg}$ on postoperative day 4, associated with a hyphema. Another eye in this group was found to have consistently elevated pressures in the 30 s secondary to development of neovascular glaucoma, leading to implantation of an Ahmed valve 4 months after surgery. One silicone oil eye with elevated postoperative day-1 pressure was found to have a hemorrhagic choroidal effusion.

Two eyes were hypotonous (IOP $\leq 5 \mathrm{~mm} \mathrm{Hg}$ ) on postoperative day 1 ( 1 with $\mathrm{SF}_{6}, 1$ with BSS). For both eyes the hypotony self-resolved during the first postoperative week, without further sequelae.

In eyes with $\mathrm{SF}_{6}$ tamponade, primary reattachment of the retina was achieved in 20 eyes (91\%); of the two eyes that re-detached, one was successfully repaired while the other was not re-operated on secondary to patient preference and severe retinal ischemia from diabetic retinopathy, as aforementioned. In this group, two eyes $(9.1 \%)$ developed a cataract requiring surgery, eight eyes (36.3\%) developed an ERM (one eye required a membranectomy), and four $(18.2 \%)$ had recurrent vitreous hemorrhage (no eyes required reoperation for this). These outcomes, including final BCVA for reoperated eyes, are shown in Table 4.

In eyes with $\mathrm{C} 3 \mathrm{~F} 8$ gas, primary reattachment was achieved in 18 eyes (82\%); all four eyes that had redetachments were successfully repaired with subsequent surgery (five total surgeries for the four eyes). Eight eyes (36.4\%) developed a visually significant cataract necessitating removal, three (13.6\%) developed an ERM (one required surgery), and five developed recurrent vitreous hemorrhage (one requiring surgery).

All 21 eyes with BSS tamponade had primary reattachment. Three eyes (14.\%) developed cataract with 


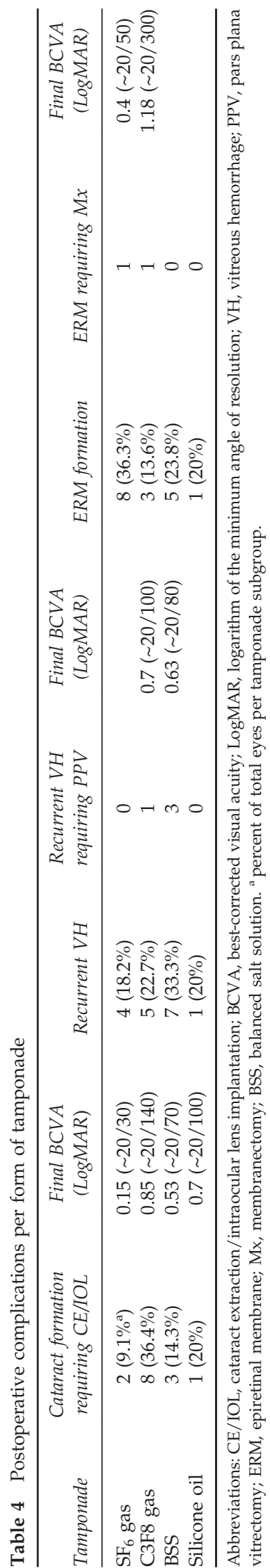

subsequent extraction, five (23.8\%) developed an ERM (none needing surgery by the conclusion of this study), and seven $(33.3 \%)$ had recurrent vitreous hemorrhage (three requiring reoperation).

Of the five eyes with silicone oil tamponade, primary reattachment was achieved in four $(80 \%)$, and the eye that redetached was successfully repaired with one additional surgery. Two eyes in this group had a combined lensectomy with primary surgery, and an additional eye developed a visually significant cataract requiring surgery. One eye in this group developed an ERM, and one had a recurrent vitreous hemorrhage (neither with further surgery).

At their latest visit, four eyes had no light perception vision but were attached anatomically; preoperative visual acuity was hand motion for these eyes, and they had progressive visual loss secondary to retinal ischemia and neovascular glaucoma.

There were no cases of postoperative endophthalmitis observed during this study.

\section{Discussion}

Use of 25-G PPV is gaining popularity in the treatment of a wide range of surgical vitreoretinal conditions. In regard to complicated TRD repair, 25-G PPV may offer several theoretical benefits over larger gauge systems. Smaller gauge PPV has more stable fluidics from diminished flow (smaller radius of 25-G tubing), which, with high cut rates, may minimize the risk of iatrogenic retinal breaks. ${ }^{16}$ Breaks may also be avoided via smaller microcannulas, as they may better preserve the vitreous base and prevent vitreal incarceration and dragging. ${ }^{10}$ Twenty-five-gauge instrumentation also allows easier access to small areas, providing safer vitrectomy and more precise dissection of membranes. 12,16

Still, there remain documented concerns with the use of 25-G PPV for complicated surgical cases, including diabetic TRD. Some worry that the flexibility of $25-G$ instrumentation may prevent adequate rotation of the eye, limiting the removal of peripheral vitreous or preretinal diabetic membranes and ultimately leading to recurrent detachments. ${ }^{12,16}$ However, stiffer instrumentation is now available. In our series, redetachment rates were low, as 63 of 70 eyes $(90 \%)$ achieved primary reattachment and final anatomical success was achieved in 69 of 70 eyes (99\%).

Another concern with (25G) sutureless PPV surgery is postoperative hypotony (here defined as IOP $\leq 5 \mathrm{~mm} \mathrm{Hg}) \cdot{ }^{16}$ In a study by Bamonte et al, ${ }^{22} \sim 9.2 \%$ of eyes were hypotonous 1 day after primary $25-\mathrm{G}$ vitreoretinal surgery; higher rates were incidentally found with fluid tamponades. ${ }^{22,23}$ In our study, two eyes were hypotonous on postoperative day 1-one eye with SF6 tamponade and the other with BSS; the hypotony 
resolved within 1 week without further intervention. It has been reported that $0.5-\mathrm{mm} 25-\mathrm{G}$ sclerotomy wounds heal more rapidly than $20-G$ wounds, ${ }^{16,24}$ perhaps supporting our observation that post 25-G PPV hypotony can self-resolve within a few days to a week. ${ }^{12,16}$

Nonetheless, hypotony is a serious concern and must be closely monitored as it can lead to choroidal detachment, escape of gas, and inadequate tamponade, ${ }^{12}$ or increased susceptibility to infection. ${ }^{22}$ To prophylactically prevent hypotony or other sequelae of would-be sutureless wounds, all sclerotomies in our series were sewn. Of note, those who prefer to leave sclerotomies sutureless have advocated hyoptony-preventative techniques such as oblique sclerotomy incisions, ${ }^{16,25}$ conjunctival displacement prior to cannula insertion, or fluid-air/gas exchange at the completion of the case. ${ }^{16}$

Increased risk of endophthalmitis with $25-\mathrm{G}$ surgery has been also been noted, presumably from direct inoculation of conjunctival bacteria during trocar insertion, decreased infusion rates during vitrectomy, and sutureless sclerotomies. ${ }^{16,22}$ In a large series, comparing endophthalmitis rates from 20- and 25-G vitrectomy surgeries, Kunimoto and Kaiser $^{26}$ found a statistically significant increase in infection rates $(0.018 \%$ vs $0.23 \%$ ) in those undergoing $25-\mathrm{G}$ vitrectomy.

However, many other large series have failed to find a such a difference. ${ }^{27-29}$ It is still imperative to undertake measures to avoid this critical complication by either tunnelling or angling the entry with the trocar or by suturing the sclerotomy site at the end of surgery. In our series, a sclerotomies were sutured. No eyes developed endophthalmitis in this study, and other complications with 25-G PPV in this study were relatively rare (Table 3).

Given the aforementioned benefits of 25-G PPV, studies have evaluated its use in diabetic TRD repair. Most studies were limited in size, retrospective, and utilized primarily silicone oil. ${ }^{6,7,17-19}$ However, a prospective, randomized study by Kumar and colleagues compared the visual and anatomic success rates of 23-G versus 25-G PPV with C3F8 or silicone oil tamponade for repair of diabetic TRD (25 eyes in both groups). Complication rates were similar, and anatomic success was achieved in all 50 eyes in the study. ${ }^{20}$ Our study also demonstrates favorable outcomes of 25-G $\mathrm{PPV}$, yet with a wider range of tamponades- $\mathrm{SF}_{6}$, C3F8, silicone oil, or BSS. Many eyes in our series had macula off detachments, combined rhegmatogenous detachments, or PVR, yet anatomic success was high, BCVA greatly improved for most patients, and complication rates were low. The few eyes that were tamponaded with silicone oil (5 out of 70) in this study had extensive and complicated detachments, and even though statistical significance for postoperative BCVA was not seen in this series (possibly because of a small number of patients in this group), they still realized significant improvements in central visual acuity. We believe this to be clinically significant.

While strengths of this study include a large population of eyes with diabetic TRD operated on by a single surgeon at a single institution, there are limitations. Postoperative acuity results were confounded by many variablesdifferences in the amount of preoperative vitreous hemorrhage, chronicity of the RD, degree of PDR and retinal vascular disease, worsening of preexisting glaucoma, and development or removal of a cataract. Conclusions of BCVA outcomes per type of tamponade may also be limited by patient selection bias secondary to location and severity of retinal detachment. Most importantly, however, the focus of this study on 25-G PPV does not allow for a direct comparison with other systems. Still, the results of this study suggest that 25-G PPV with $\mathrm{SF}_{6}, \mathrm{C} 3 \mathrm{~F} 8$, silicone oil, or BSS is a safe and effective modality for the treatment of complicated diabetic TRD.

\section{Summary}

What was known before

- Twenty-five-gauge pars plana vitrectomy offers many benefits over larger gauge systems-namely reduced operative times, reduced post-operative inflammation, faster visual recovery, increased patient comfort, reduced rate of intraoperative retinal break formation, avoidance of conjunctival dissection and limbal stem cell damage, and less induced corneal astigmatism.

- Several small studies have investigated the use of 25-G vitrectomy for complicated retinal detachment, including diabetic tractional retinal detachment (TRD); comparable outcomes and complication rates between 25-G PPV (with silicone oil or octofluoropropane only) and $20-\mathrm{G}$ or $23-\mathrm{G}$ systems have been found.

\section{What this study adds}

- In our large series, 25-gauge vitrectomy was effective and safe in the repair of diabetic tractional retinal detachment, including surgically complicated cases of combined tractional and rhegmatogenous detachments or proliferative vitreoretinopathy.

- Sulfur hexafluoride, octofluoropropane, silicone oil, or balanced salt solution may all be safely and effectively used as tamponade agents in 25-gauge repair of diabetic TRD.

\section{Conflict of interest}

JIL has commercial relationship with QLT (F), Genentech $(\mathrm{R})$, and Regeneron $(\mathrm{R})$. The remaining authors declare no conflict of interest. 


\section{References}

1 Rice TA, Michels RG, Rice EF. Vitrectomy for diabetic traction retinal detachment involving the macula. Am J Ophthalmol 1983; 95: 22-33.

2 Fong DS, Ferris 3rd FL, Davis MD, Chew EY. Causes of severe visual loss in the early treatment diabetic retinopathy study: ETDRS report no. 24. Early Treatment Diabetic Retinopathy Study Research Group. Am J Ophthalmol 1999; 127: 137-141.

3 Regler R, Sachs HG, Hillenkamp J, Helbig H, Framme C. Long-term evaluation of anatomic and functional results after complicated retinal detachment treated with pars plana vitrectomy and heavy silicone oil tamponade. Klin Monbl Augenheilkd 2009; 226: 707-712.

4 Kirchhof B. Strategies to influence PVR development. Graefes Arch Clin Exp Ophthalmol 2004; 242: 699-703.

5 Fujii GY, de Juan Jr E, Humayun MS, Pieramici DJ, Chang TS, Awh C et al. A new 25-gauge instrument system for transconjunctival sutureless vitrectomy surgery. Ophthalmology 2002; 109: 1807-1812.

6 Lakhanpal RR, Humayun MS, de Juan Jr E, Lim JI, Chong LP, Chang TS et al. Outcomes of 140 consecutive cases of 25-gauge transconjunctival surgery for posterior segment disease. Ophthalmology 2005; 112: 817-824.

7 Sato T, Emi K, Bando H, Ikeda T. Faster recovery after 25 gauge microincisionvitrectomy surgery than after 20-gauge vitrectomy in patients with proliferative diabetic retinopathy. Clin Ophthalmol 2012; 6: 1925-1930.

8 Narayanan R, Sinha A, Reddy RK, Krishnaiah S, Kupperman BD. Faster visual recovery after 23-gauge vitrectomy compared with 20-gauge vitrectomy. Retina 2010; 30: 1511-1514

9 Rizzo S, Genovesi-Ebert F, Murri S, Belting C, Vento A, Cresti $\mathrm{F}$ et al. 25-gauge, sutureless vitrectomy and standard 20-gauge pars plana vitrectomy in idiopathic epiretinal membrane surgery: a comparative pilot study. Graefes Arch Clin Exp Ophthalmol 2006; 244: 472-479.

10 Neuhann IM, Hilgers RD, Bartz-Schmidt KU. Intraoperative retinal break formation in 23-/25-gauge vitrectomy versus 20-gauge vitrectomy. Ophthalmologica 2013; 229: 50-53.

11 de Paula AC, Nassaralla Junior JJ, Rodrigues FW, da Silva RE, Correa e Silva RS, Barbosa Junior DR. Alterations in the corneal topography after pars plana vitrectomy with the standard system of 20-gauge and the 25-gauge transconjunctival sutureless vitrectomy. Semin Ophthalmol 2012; 27: 11-14

12 Thompson JT. Advantages and Limitations of Small Gauge Vitrectomy. Surv Ophthalmol 2011; 56: 162-172.

13 Ibarra MS, Hermel M, Prenner JL, Hassan TS. Longer-term outcomes of transconjunctival sutureless 25-gauge vitrectomy. Am J Ophthalmol 2005; 139: 831-836.

14 Inoue M, Noda K, Ishida S, Nagai N, Imamura Y, Oguchi Y. Intraoperative breakage of a 25-gauge vitreous cutter. Am J Ophthalmol 2004; 138: 867-869.
15 Byeon SH, Chu YK, Lee SC, Koh HJ, Kim SS, Kwon OW. Problems associated with the 25-gauge transconjunctival sutureless vitrectomy system during and after surgery. Ophthalmologica 2006; 220: 259-265.

16 Chen E. 25-Gauge transconjunctival sutureless vitrectomy. Curr Opin Ophthalmol 2007; 18: 188-193.

17 Shah CP, Ho AC, Regillo CD, Fineman MS, Vander JF, Brown GC. Short-term outcomes of 25-gauge vitrectomy with silicone oil for repair of complicated retinal detachment. Retina 2008; 28: 723-728.

18 Altan T, Acar N, Kapran Z, Unver YB, Ozdogan S. Transconjunctival 25-gauge sutureless vitrectomy and silicone oil injection in diabetic tractional retinal detachment. Retina 2008; 28: 1201-1206.

19 Park KH, Woo SJ, Hwang JM, Kim JH, Yu YS, Chung H. Short-term outcome of bimanual 23-gauge transconjunctival sutureless vitrectomy for patients with complicated vitreoretinopathies. Ophthalmic Surg Lasers Imaging 2010; 41: 207-214.

20 Kumar A, Duraipandi K, Gogia V, Sehra SV, Gupta S, Midha N. Comparative evaluation of 23- and 25-gauge microincision vitrectomy surgery in management of diabetic macular traction retinal detachment. Eur J Ophthalmol 2014; 24: 107-113.

21 Holladay JT. Visual acuity measurements. J Cataract Refract Surg 2004; 30: 287-290.

22 Bamonte G, Mura M, Stevie Tan H. Hypotony After 25-Gauge Vitrectomy. Am J Ophthalmol 2011; 151: 156-160.

23 Gupta OP, Weichel ED, Regillo CD, Fineman MS, Kaiser RS, Ho AC et al. Postoperative complications associated with 25-guage pars plana vitrectomy. Ophthalmic Surg Lasers Imaging 2007; 38: 270-275.

24 Keshavamurthy R, Ventkatesh P, Garg S. Ultrasound biomicroscopy findings of $25 \mathrm{G}$ transconjunctival sutureless (TSV) and conventional (20G) pars plana sclerotomy in the same patient. BMC Ophthalmol 2006; 6: 7.

25 Hsu J, Chen E, Gupta O, Fineman MS, Garg SJ, Regillo CD Hypotony after 25-gauge vitrectomy using oblique versus direct cannula insertions in fluid-filled eyes. Retina 2008; 28: 937-940.

26 Kunimoto DY, Kaiser RS. Incidence of endophthalmitis after 20- and 25-gauge vitrectomy. Ophthalmology 2007; 114: 2133-2137.

27 Hu AY, Bourges JL, Shah SP, Gupta A, Gonzales CR, Oliver $\mathrm{SC}$ et al. Endophthalmitis after pars plana vitrectomy: a 20- and 25-gauge comparison. Ophthalmology 2009; 116: 1360-1365.

28 Scott IU, Flynn Jr HW, Dev S, Shaikh S, Mittra RA, Arevalo JF et al. Endophthalmitis after 25-gauge and 20-gauge pars plana vitrectomy: incidence and outcomes. Retina 2008; 28: 138-142.

29 Mason 3rd JO, Yunker JJ, Vail RS, White Jr MF, Feist RM, Thomley ML et al. Incidence of endophthalmitis following 20-gauge and 25-gauge vitrectomy. Retina 2008; 28: 1352-1354. 